\title{
Uma Comparação do Poder Preditivo de Diferentes Variáveis para Períodos de Recessão da Economia Brasileira
}

\section{A Comparison of the Predictive Power of Different Variables for Periods of Recession in the Brazilian Economy}

\author{
Vitor Fernandes Cavalcante ${ }^{\mathrm{a}, \mathrm{b}}$ \\ Heleno Piazentini Vieirac
}

\begin{abstract}
Resumo: Este trabalho busca comparar o poder preditivo de diferentes variáveis nas recessões datadas para a economia brasileira no período de 1997 a 2017 . O modelo econométrico utilizado é um probit simples que fornece a probabilidade de a economia brasileira entrar em recessão de um a oito trimestres à frente. Com isso, é possível construir o pseudo $\mathrm{R}^{2}$, o qual permite comparar a performance de diferentes variáveis para cada horizonte de previsão. Dentre os resultados encontrados, destacam-se, para previsões de curto prazo, a utilização de variáveis que captam a situação fiscal do país, o índice de preços internacional das commodities e variáveis financeiras, como o retorno do índice Ibovespa e o risco-país. Entretanto, o trabalho identifica um gap na literatura referente a variáveis que possam prever recessões para horizontes de tempo mais distantes.
\end{abstract}

Palavras-chave: Pseudo $R^{2}$. Recessão. Brasil. Probit.

\begin{abstract}
This paper seeks to compare the predictive power of different variables during periods of recession for the Brazilian economy from 1997 until 2017. The econometric model used is the simple probit that provides the probability of the economy going into recession from one to eight quarters ahead. The model makes it possible to estimate the pseudo $\mathrm{R}^{2}$ as a parameter of comparison between the predictive power of the variables analyzed. Among the results found, the use of variables that capture the country's fiscal situation, the international commodity price index and financial variables, such as the return of the Ibovespa index and the country risk stand out for shortterm forecasts. However, this article identifies a gap in the Brazilian literature regarding variables that can forecast recessions for more distant time horizons.
\end{abstract}

Keywords: Pseudo $R^{2}$. Recession. Brazil. Probit.

JEL Classification: E32; E37; C53; N16.

\footnotetext{
a Universidade Nova de Lisboa, Nova School of Business and Economics, NOVAFRICA. Lisboa, Portugal.

b Insper, Centro de Gestão e Políticas Públicas (CGPP), Cátedra Ruth Cardoso. São Paulo, São Paulo, Brasil.

c Insper. São Paulo, São Paulo, Brasil.
} 


\section{1 lntrodução}

A previsão de períodos de recessão de uma economia é de suma importância para o processo de tomada de decisão das famílias, empresas e instituições governamentais. Quanto mais cedo for detectado o movimento de contração econômica, mais rápidas serão as reações dos governantes, a fim de minimizar os efeitos de uma crise, dos investidores, ao remanejar seus investimentos em busca do maior retorno, e das famílias, que moldam suas decisões buscando uma suavização do consumo ao longo do tempo (GRUBER, 2013).

Diante disso, diversos estudos empíricos, desde a década de 1990, buscam estabelecer relações entre os períodos de recessão de uma economia com variáveis que potencialmente teriam a capacidade de prever esse movimento. Uma das alternativas, proposta primeiramente por Estrella e Hardouvelis (1991) e aprofundada posteriormente por Estrella e Mishkin (1998), é construir modelos que preveem a probabilidade de desaceleração econômica através de variáveis respostas binárias.

Estrella e Mishkin (1998) constroem um pseudo $R^{2}$ a fim de identificar quais potenciais variáveis apresentam o maior poder preditivo para períodos de recessão da economia norte-americana. Assim, comparam o poder preditivo de diversas variáveis financeiras, como os agregados monetários, o índice da bolsa de valores de Nova Iorque (Nyse) e o spread entre as taxas de juros de curto e longo prazos do governo norte-americano. Todos foram estatisticamente relevantes para prever períodos de recessão, sendo o spread, entre as taxas de juros, a que apresenta o maior poder preditivo.

Desde, então, vários trabalhos acadêmicos tentam identificar relações semelhantes para outros países utilizando o modelo proposto por Estrella e Mishkin (1998), ou seja, um probit, nas suas mais diversas variações. Dentre eles, podemos destacar os de Chauvet e Potter (2005), Chauvet e Morais (2010), Hao e Ng (2011) e Bismans e Majetti (2013).

No trabalho sobre a economia brasileira, Chauvet e Morais (2010) têm como objetivo comparar a capacidade preditiva de modelos probit compostos por mais de uma variável explicativa, utilizando variáveis que refletem as políticas do governo, as mudanças no processo produtivo do país e as expectativas dos agentes econômicos. Além disso, o trabalho identifica uma superioridade do modelo probit dinâmico em relação ao simples. O probit dinâmico não restringe a variação dos parâmetros do modelo ao longo do tempo e possui a variável latente como fator autorregressivo.

Oliveira (2016), assim como Chauvet e Morais (2010), busca encontrar modelos com mais de uma variável explicativa que possam prever períodos de recessão da economia brasileira, focando na utilização de variáveis financeiras e do setor real da economia e utilizando como método comparativo entre modelos a 
função receiver operating characteristic (ROC). Assim como o presente artigo, Oliveira (2016) faz uso do retorno do índice de ações do mercado brasileiro, da taxa de juros nominal e da oferta monetária M2 como variáveis de análise.

Ainda no contexto brasileiro, Duarte, Issler e Spacov (2004) apresentam indicadores coincidentes para períodos de recessões. Para auxiliar a construção do índice, foram utilizadas séries antecedentes submetidas ao teste de causalidade Granger com séries coincidentes. As séries antecedentes encontradas pelos autores que também foram analisas neste artigo são: a taxa de câmbio com relação ao dólar, a taxa de juros nominal e o Ibovespa.

O interesse deste trabalho está na comparação e na seleção das variáveis potenciais que possam compor tanto modelos dinâmicos quanto simples, tendo o foco na construção do pseudo $R^{2}$, proposto por Estrella e Mishkin (1998), para diferentes variáveis da economia brasileira. Dessa forma, a utilização do modelo probit simples, sem a presença da variável latente como fator autorregressivo, permite identificar a capacidade que uma variável de interesse isoladamente possui para prever períodos de recessão. Portanto, o foco deste estudo não é encontrar a melhor combinação de variáveis que prevê períodos de recessão, como proposto por outros trabalhos no contexto brasileiro, mas identificar quais são as variáveis que isoladamente apresentam maior poder preditivo para o curto e médio prazos, de modo a prover um critério de escolha para a construção de modelos probit . Desse modo, tanto a estimação de modelos dinâmicos, quanto a utilização da função ROC não adicionaria a este artigo informações relevantes para a capacidade preditiva das variáveis isoladamente, uma vez que esses métodos são comumente usados para avalição de modelos com mais de uma variável explicativa, mostrando a capacidade preditiva da combinação de diferentes variáveis.

Assim como o artigo de Estrella e Mishkin (1998), o pseudo $R^{2}$ é calculado através dos métodos in sample e out of sample. O método in sample utiliza todos os dados da amostra, e os valores estimados são comparados com os valores observados. Já a análise out of sample é feita primeiramente estimando o modelo do começo da amostra até determinada data, por exemplo, o primeiro trimestre de 2008. Essa estimação é utilizada para fazer projeções para trimestres posteriores, a título de exemplo, dois trimestres à frente. Assim, pode-se obter o valor previsto para o terceiro trimestre de 2008. Após a adição de mais um valor amostral, referente ao segundo trimestre de 2008 , é possível projetar o valor para o quarto trimestre de 2008. O processo é repetido inúmeras vezes, permitindo a construção de uma série que seria a prevista para uma dada quantidade de informação passada existente naquele período de tempo.

O método out of sample permite uma análise mais realista da capacidade preditiva das diferentes variáveis em comparação com o in sample. Entretanto, esse tipo de estimativa possui algumas desvantagens, apontadas por Estrella e Mishkin (1998). Primeiramente, uma regressão diferente deve ser estimada para a projeção 
de cada trimestre subsequente. Além disso, não é mais garantido que os valores do pseudo $R^{2}$ encontrados estejam entre zero e um, e, caso isso ocorra, não é possível que uma interpretação seja feita. Portanto, o teste de significância perde sentido com o método out of sample.

O período abordado vai desde o primeiro trimestre de 1997 até o quarto trimestre de 2017. A escolha do período é baseada em dois aspectos principais. O primeiro deles é o fato de a economia brasileira ter passado por um longo período de instabilidade inflacionária, desde a década de 1980 até a metade da década de 1990, com o programa de estabilização do Plano Real. O artigo de Lopes, Chauvet e Lima (2017) mostra evidências estatísticas de que a volatilidade da economia brasileira cai significativamente após o plano de estabilização. Também, o ano de 1996 ainda apresenta um ambiente de estabilização em processo de consolidação. Sendo assim, a inclusão do período anterior a 1997 na amostra pode potencialmente distorcer os resultados do modelo estimado.

O segundo aspecto é a periodicidade de publicação da datação dos períodos de recessão, divulgadas pelo Comitê de Datação de Ciclos Econômicos (Codace). O órgão não possui um prazo definido para publicação de seus relatórios. No último trabalho publicado, em outubro de 2017, o Codace realizou a datação dos períodos de recessão e expansão econômicos até o quarto trimestre de 2016 (FGV, 2017). Como as publicações ocorrem apenas quando há uma quebra no período de recessão ou crescimento, este trabalho considera expansionista os trimestres do ano de 2017.

Este artigo está organizado da seguinte forma: a segunda seção apresenta a metodologia utilizada, detalhando o modelo econométrico, o critério de datação dos períodos de recessão da economia brasileira segundo o Codace e a seleção das variáveis explicativas; a terceira seção trata dos resultados encontrados; a quarta seção apresenta as considerações finais da pesquisa.

\section{Modelo Probit}

O modelo utilizado para avaliar a qualidade das variáveis selecionadas em prever períodos de recessão é o probit, na sua versão mais simples, seguindo a abordagem proposta por Estrella e Mishkin (1998).

Diferentemente do modelo dinâmico proposto por Chauvet e Morais (2010), a versão simples restringe a variação dos parâmetros estimados ao longo do tempo e, além disso, não conta com um processo autorregressivo para variável latente. $\mathrm{O}$ trabalho de Chauvet e Morais (2010) mostra uma melhor performance do modelo dinâmico em relação ao simples, estimando a probabilidade de recessão para a economia brasileira do primeiro trimestre de 1995 até o último de 2007. Entretanto, este trabalho busca, na restrição da variação dos parâmetros ao longo do tempo, a construção do pseudo $R^{2}$, como no trabalho de Estrella e Mishkin (1998), 
permitindo comparar o poder preditivo das diferentes variáveis analisadas isoladamente. Dessa forma, o trabalho provê um critério de escolha para a construção de modelos probit nas suas mais variadas formas.

O modelo simples tem como premissa que a variável de interesse tenha comportamento binário, ou seja, a variável pode assumir apenas dois valores: um, para períodos de recessão, e zero, caso o contrário. O modelo é definido pela seguinte relação expressa:

$$
Y_{\mathrm{t}+\mathrm{k}}=\beta^{\prime} X_{\mathrm{t}}+E_{\mathrm{t}}
$$

na qual Yt é a variável não observada, que representa o estado da economia no período $t ; k$ é o horizonte de tempo para o qual será feita a previsão; $\beta$ é o vetor de coeficientes; $X_{\mathrm{t}}^{\mathrm{e}}$ o vetor de variáveis explicativas, incluindo uma constante; e $E_{\mathrm{t}}$ é o termo de erro, que segue uma distribuição normal.

O indicador observável, $R_{\mathrm{t}}$, que representa a datação dos períodos de recessão, se relaciona com o modelo pela expressão:

$$
\begin{aligned}
& R_{\mathrm{t}}=1, \text { se } Y_{\mathrm{t}} \geq 0 \\
& R_{\mathrm{t}}=0 \text { se } Y_{\mathrm{t}}<0
\end{aligned}
$$

Dessa forma, a probabilidade de a economia estar em recessão $k$ trimestres à frente é função da toda informação disponível em $t$, referente ao vetor de variáveis independentes $X_{\mathrm{t}}$. Assim, tem-se que:

$$
P\left(R_{\mathrm{t}+\mathrm{k}}=1\right)=F\left(\beta^{\prime} X_{\mathrm{t}}\right)
$$

em que $F$ representa a função de distribuição normal correspondente ao termo de erro $E_{\mathrm{t}}$. $O$ modelo é estimado pelo método de máxima verossimilhança, com a função de verossimilhança definida por:

$$
\begin{gathered}
L=\prod F\left(\beta^{\prime} X_{\mathrm{t}}\right) \prod\left[1-F\left(\beta^{\prime} X_{\mathrm{t}}\right)\right] \\
\left(R_{\mathrm{t}+\mathrm{k}}=1\right) \quad\left(R_{\mathrm{t}+\mathrm{k}}=0\right)
\end{gathered}
$$

Através da função de máxima verossimilhança, é possível obter as funções logarítmicas de máxima verossimilhança:

$$
\ln L=\sum_{i=1}^{N}\left[y_{i} \ln F\left(X^{\prime}{ }_{i} \beta\right)+\left(1-y_{i}\right) \ln \left(1-F\left(X_{i}^{\prime} \beta\right)\right)\right]
$$

Dessa forma, pode-se calcular o valor do pseudo $R^{2}$ proposto por Estrella e Mishkin (1998), definido por:

$$
\text { Pseudo } \mathrm{R}^{2}=1-(\log \mathrm{Lu} / \log \mathrm{Lc})^{-(2 / \mathrm{n}) \log \mathrm{Lc}}
$$


em que $n$ é o número de observações na amostra, $L u$ é o $L$ da função de máxima verossimilhança do modelo irrestrito e $L c$ é o valor da função de máxima verossimilhança para o modelo restrito. Este possui a restrição de que todas as variáveis explicativas são insignificantes estatisticamente, com exceção da constante.

Um pseudo $R^{2}$ mais alto sugere que as variáveis explicativas são mais relevantes, pois aumentam a função de verossimilhança em comparação com o modelo com apenas uma constante. O método é aplicado para um horizonte de tempo até oito trimestres à frente. Dessa forma, é possível estabelecer a capacidade preditiva da variável no curto e médio prazos. Porém, vale notar que o pseudo $R^{2}$ não possui a mesma interpretação do $R^{2}$ tradicional da econometria, portanto o resultado não mostra o quanto da variação da variável explicativa está sendo explicada pelas variáveis independentes.

O pacote econométrico utilizado para estimar o modelo é o Eviews, em sua versão 9.0. Além disso, com exceção da datação dos períodos de recessão, do índice de preços internacional das commodities e do risco país, todas as variáveis apresentadas neste trabalho foram extraídas do Instituto de Pesquisa Econômica Aplicada (Ipea) (BRASIL, [2018]).

\subsection{As Recessões Datadas e a Seleção das Variáveis Explicativas}

Para a construção da variável binária, que representa períodos de recessão ou expansão da economia brasileira, são utilizados dados do Codace, criado pelo Instituto Brasileiro de Economia (Ibre) da Fundação Getúlio Vargas (FGV). O comitê considera:

[...] como recessão os períodos em que houve expressivo declínio no nível de atividade econômica espalhado por diversos segmentos da economia brasileira, perceptível durante ao menos dois trimestres consecutivos. A principal variável usada na datação foi o Produto Interno Bruto (PIB) trimestral, a preços de mercado, dessazonalizado, calculado pelo IBGE. Para confirmar os pontos de transição detectados na série de PIB trimestral, o comitê utilizou todas as principais séries econômicas disponíveis, principalmente aquelas que melhor expressavam, no respectivo período, indicações do estado da produção, vendas, emprego e renda na economia brasileira (FGV, 2017).

O Gráfico 1 mostra a datação do Codace paro o período abordado neste trabalho e o crescimento real do PIB brasileiro: 
Gráfico 1 - Períodos de recessão da economia brasileira e o crescimento do PIB

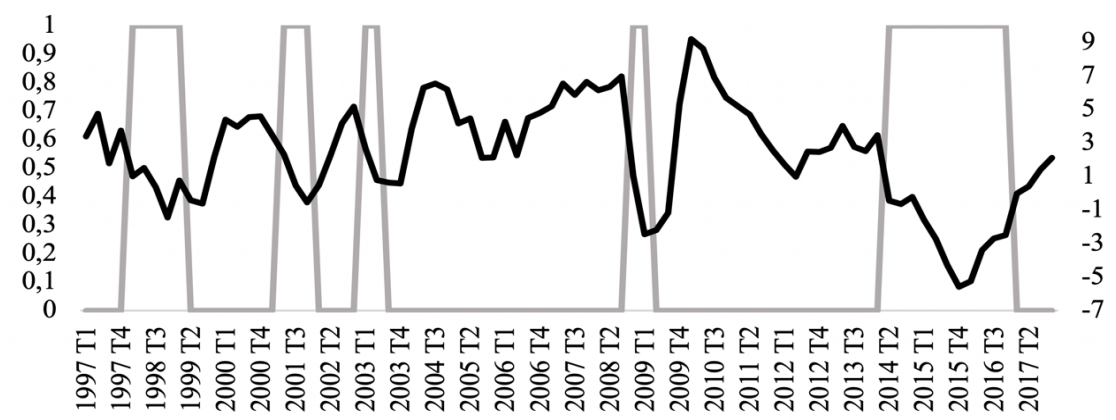

Recessões Crescimento Real - PIB - Trimestral (Lado Direito)

Fonte: Codace (FGV, 2017)

Durante o período abordado, o Brasil passou por cinco períodos de recessão, sendo o último, do segundo trimestre de 2014 até o quarto trimestre de 2016, o mais longo. As causas das recessões variam de acordo com o contexto histórico e as decisões governamentais do país.

O cenário internacional, por exemplo, ajuda a explicar as recessões brasileiras datadas em contexto de arranjo cambial fixo, a partir dos efeitos das crises asiáticas e russa no final da década de 1990 (PASTORE; PINOTTI, 1999). As crises externas foram importantes para explicar períodos recessivos também a partir da flutuação cambial, como nos casos da crise da Argentina, dos atentados terroristas em 2001 e da crise financeira mundial em 2008 (GIAMBIAGI et al., 2011).

Já no âmbito doméstico, o cenário eleitoral de 2002, que trouxe forte elevação do risco-país, ajuda a explicar a recessão do início de 2003 (ABREU, 2015). Da mesma forma, as escolhas de política econômica a partir de 2011, como, por exemplo, a reversão da política monetária, que desorganizou as expectativas inflacionárias (MESQUITA, 2014), ajudam a explicar a última recessão datada.

A seleção das variáveis explicativas com poder preditivo de trimestres de recessão é baseada nos trabalhos de Chauvet e Morais (2010) e Estrella e Mishkin (1998), que envolvem a classificação dos principais indicadores em quatro categorias principais:

a) medidas de fases iniciais do processo produtivo;

b) medidas que refletem a flexibilidade do processo produtivo;

c) séries que refletem as políticas fiscal e monetária do governo;

d) proxies de expectativas relacionadas ao mercado e a possíveis canais de transmissão para uma crise financeira.

Assim, a partir da breve contextualização exposta, parece importante considerar um conjunto de variáveis a serem selecionadas e que são avaliadas pelo 
presente estudo: alguma medida para a evolução do preço das commodities; a evolução da taxa básica de juros (Selic); a dinâmica da taxa nominal de câmbio; uma medida do risco-país; medidas que refletem a política fiscal nos diferentes níveis governamentais; um índice representativo da evolução do mercado acionário nacional e sua volatilidade; as mudanças no nível de reservas internacionais e na oferta monetária brasileira.

\section{Resultados}

As Tabelas 1 e 2, apresentadas no Apêndice A, mostram todas as variáveis, dentre as analisadas, que se mostraram relevantes para pelo menos um dos oito horizontes de tempo em questão na análise in sample e seus respectivos pseudo $R^{2}$. O procedimento adotado segue a equação:

$$
P\left(R_{\mathrm{t}+\mathrm{k}}=1\right)=F\left(\alpha_{0}+\alpha_{1} x_{1, \mathrm{t}}\right)
$$

Assim, é possível observar se a adição da variável explicativa na equação com a constante é estatisticamente significante (no caso da análise in sample). Além disso, se é significante, qual é o poder preditivo que a adição dessa variável gera para prever períodos de recessão da economia brasileira utilizando o pseudo $R^{2}$ como medida comparativa.

Para o caso da análise out of sample, apenas os pseudo $R^{2}$ são apresentados nas Tabelas 1 e 2, uma vez que não é possível realizar o teste de significância. Para valores negativos do pseudo $R^{2}$, um traço foi utilizado para representá-los nas Tabelas 1 e 2. Já na análise gráfica presente nesta seção, valores negativos de pseudo $R^{2}$ são substituídos por zero como justificativa puramente estética.

\section{I Variáveis Destaque no Curto Prazo}

As variáveis que mostram um maior poder preditivo para horizontes de um a quatro trimestres à frente refletem a situação fiscal do país, seguidas por variáveis financeiras, que indicam as expectativas dos agentes econômicos. Outro importante resultado é encontrado a partir do índice de preço das commodities, que aparece com o maior pseudo $R^{2}$ para previsões de um trimestre à frente, porém com perda de poder preditivo significativo para horizontes de tempo mais longos.

Os Gráficos 2 e 3 mostram o pseudo $R^{2}$ das análises in sample e out of sample, respectivamente, estimado para cada horizonte de tempo analisado com variáveis que dizem respeito à situação fiscal do país. 
Gráfico 2 - Pseudo $R^{2}$ (in sample) para variáveis que refletem a situação fiscal do país

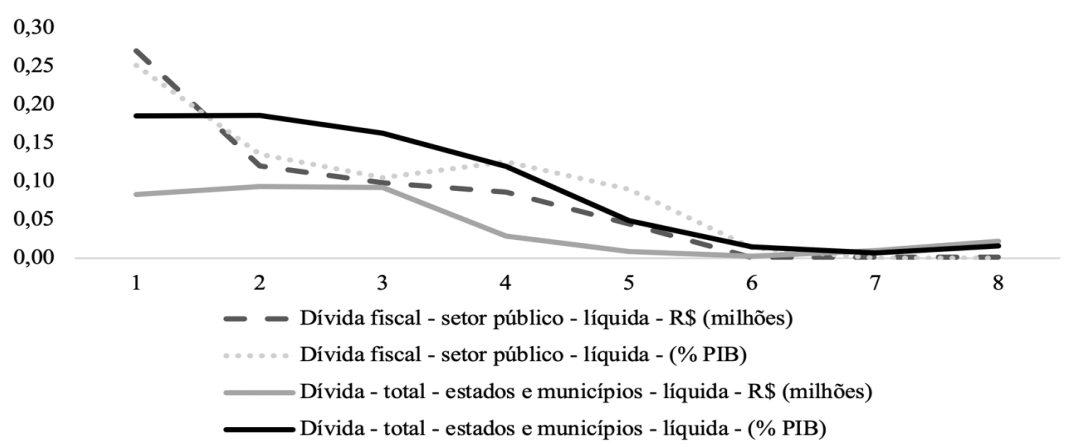

Fonte: Elaboração própria.

Gráfico 3 - Pseudo $R^{2}$ (out of sample) para variáveis que refletem a situação fiscal do país

$$
\begin{aligned}
& 0,30 \\
& 0,25 \\
& 0,20 \\
& 0,15 \\
& 0,10 \\
& 0,05 \\
& 0,00
\end{aligned}
$$

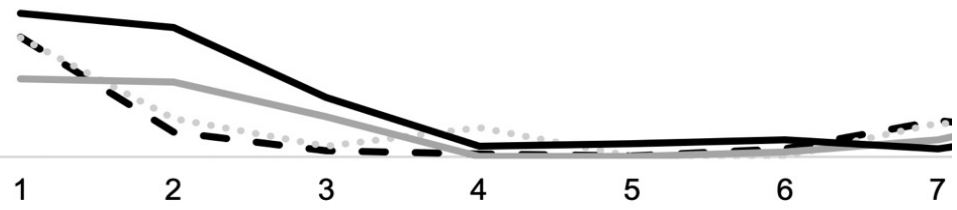

- - Dívida fiscal - setor público - líquida - $\mathrm{R} \$$ (milhões)

Dívida fiscal - setor público - líquida - (\% PIB)

Fonte: Elaboração própria.

A inclusão dessas variáveis para analisar a probabilidade de períodos de recessão é inédita e o resultado condiz com o descontrole das contas públicas enfrentado pelo país nos últimos anos. O Gráfico 4, que mostra a evolução da taxa de crescimento da dívida fiscal líquida do setor público, ilustra como o período recessivo iniciado em 2014 foi marcado pela deterioração fiscal do país. ${ }^{1}$

1 Utiliza-se a taxa de crescimento na estimação dos modelos para variáveis não estacionárias. 
Gráfico 4 - Recessões e taxa de crescimento da dívida fiscal líquida (setor público)

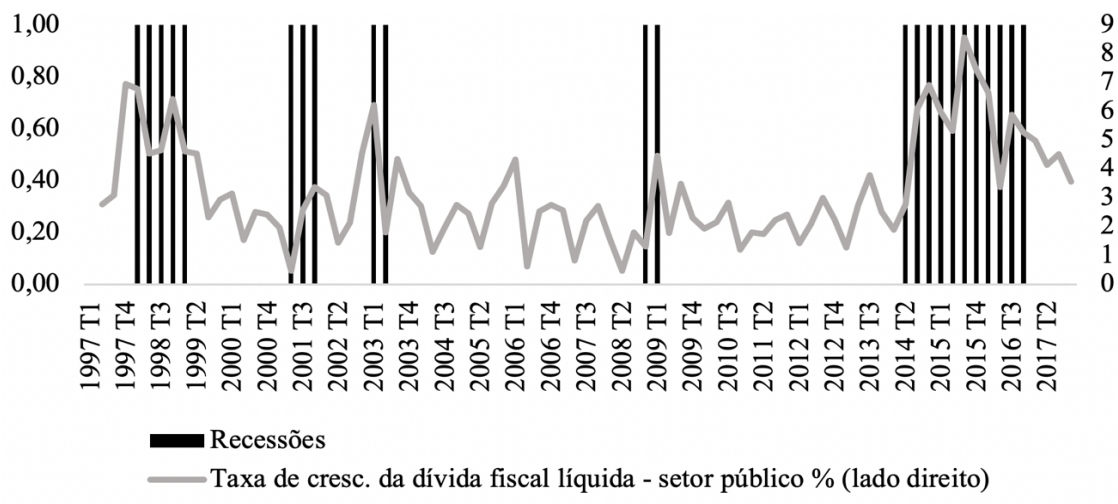

Fonte: Elaboração própria.

Vale notar que os valores encontrados para o pseudo $R^{2}$ e o poder preditivo das variáveis para diferentes horizontes de tempo possuem uma trajetória similar nas análises in sample e out of sample. Esses resultados permitem concluir que variáveis que capturam a situação fiscal do país podem ser incorporadas a modelos probit com um ou dois lags de defasagem.

No caso das variáveis financeiras, nota-se a perda do poder preditivo da taxa de juros e da variação cambial na análise out of sample em comparação com a análise in sample. Entretanto, destacam-se o retorno do índice Ibovespa e o risco-país como candidatos de curto prazo, como mostram os Gráficos 5 e 6. Esse resultado condiz com a literatura sobre o tema, uma vez que, nos trabalhos de Chauvet e Morais (2010) e Oliveira (2016), o retorno do índice Ibovespa é utilizado nos modelos de curto prazo propostos. Além disso, a variável também é utilizada como um indicador antecedente no trabalho de Duarte, Issler e Spacov (2004). Entretanto, chama a atenção a fraca performance relativa da taxa de juros Selic na análise out of sample para prever períodos de recessão, variável que foi utilizada nos modelos propostos pelos trabalhos citados. 
Gráfico 5 - Pseudo $R^{2}$ (in sample) para variáveis financeiras

0,10
0,08
0,06
0,04
0,02
0,00

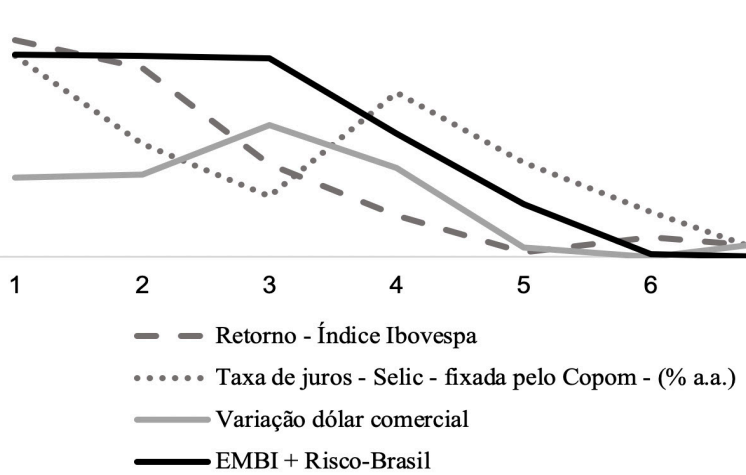

Fonte: Elaboração própria.

Gráfico 6 - Pseudo $R^{2}$ (out of sample) para variáveis financeiras

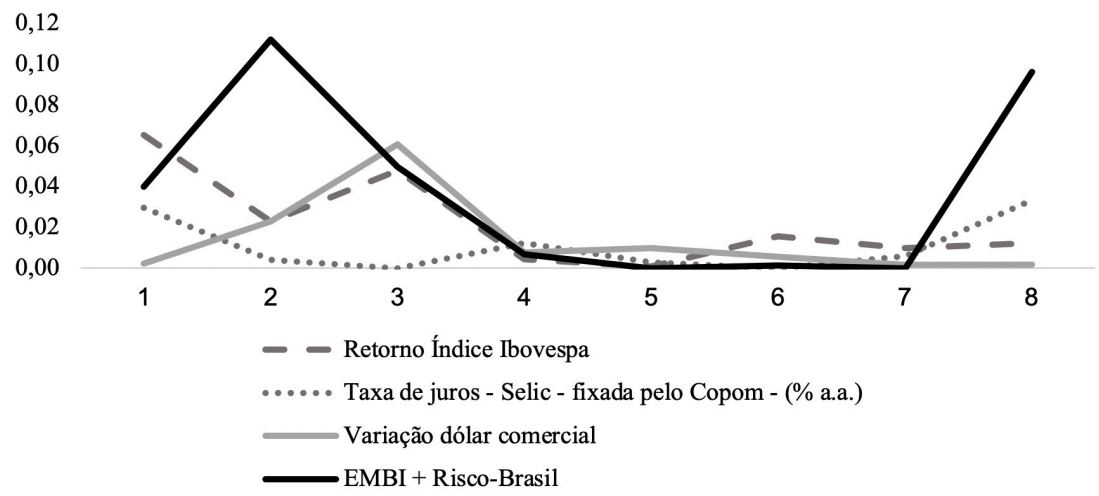

Fonte: Elaboração própria.

Além de variáveis que refletem a situação fiscal e as expectativas dos agentes econômicos, é notável a boa performance relativa de variáveis que capturam efeitos do mercado internacional na economia brasileira. Como mencionado, o indicador de preço internacional das commodities foi a variável que apresentou o maior pseudo $R^{2}$ para previsão de recessões um trimestre à frente tanto na análise in sample, quanto na análise out of sample. Além de um indicador do preço das commodities, foram testadas duas variáveis de preços relacionadas ao setor externo brasileiro, sendo elas os preços das importações e exportações brasileiras, apresentadas no Gráfico 7. Porém, nenhuma se destaca tanto quanto o índice de preço das commodities. 
Gráfico 7 - Pseudo $R^{2}$ (in sample) de variáveis de preços para o setor externo

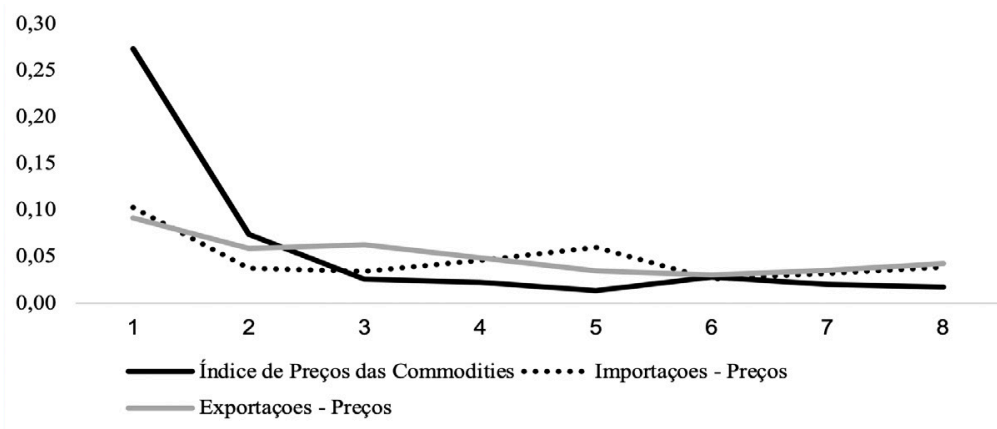

Fonte: Elaboração própria.

Gráfico 8 - Pseudo $R^{2}$ (out of sample) de variáveis de preços para o setor externo

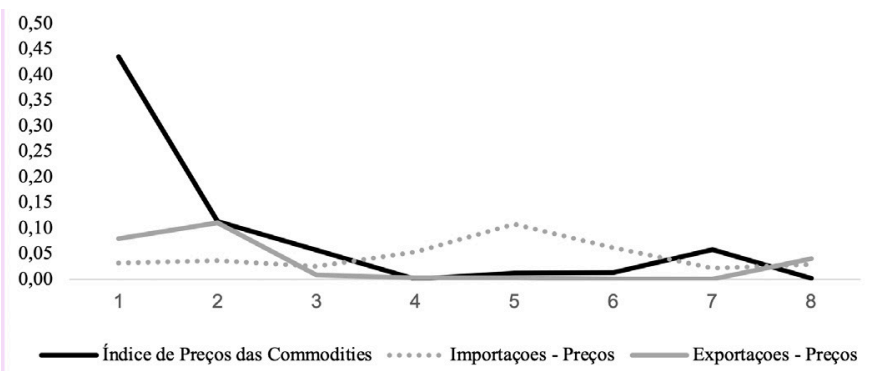

Fonte: Elaboração própria.

\subsection{Variáveis Destaque no Médio Prazo}

A literatura que aborda os modelos probit para a identificação de indicadores líderes tem como principal variável o spread entre as taxas de juros de curto e longo prazos do país. Entretanto, tal análise não é viável para a economia brasileira, uma vez que as séries de títulos do governo com vencimento em três meses e dez anos não possuem um número de observações suficientes para a estimação de modelos probit consistentes.

Dessa forma, este trabalho buscou identificar outras variáveis que possam apresentar um bom desempenho em modelos de médio prazo como forma de gerar variáveis alternativas ao spread das taxas de juros na composição de modelos probit.

Chauvet e Morais (2010) utilizam, em seus modelos probit dinâmicos de médio prazo, o consumo residencial de energia elétrica, o índice de preços das 
importações, as mudanças na balança comercial, o estoque de veículos comerciais leves produzidos, o estoque de ônibus produzidos e um índice de falências.

Este artigo propõe a análise de outras variáveis que refletem as políticas monetária e cambial do governo e uma variável financeira. Assim, são testadas a taxa de crescimento da oferta monetária, medida pelo M2 deflacionado pelo IPCA, a taxa de crescimento das reservas internacionais, utilizando-se o conceito de caixa, e a volatilidade do índice Ibovespa. Além disso, o consumo de energia elétrica é considerado, uma vez que Chauvet e Morais (2010) e Oliveira (2016) utilizam variáveis do setor energético em seus modelos de médio prazo.

Gráfico 9 - Pseudo $R^{2}$ (in sample) para variáveis destaques no médio prazo

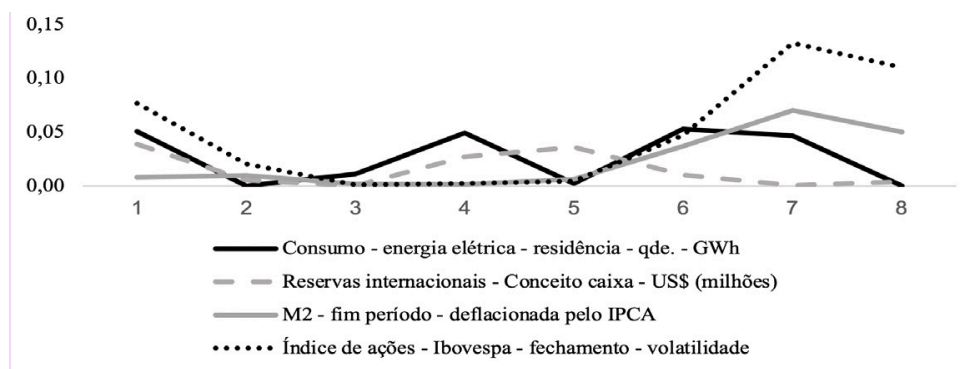

Fonte: Elaboração própria.

Gráfico 10 - Pseudo $R^{2}$ (out of sample) para variáveis destaques no médio prazo

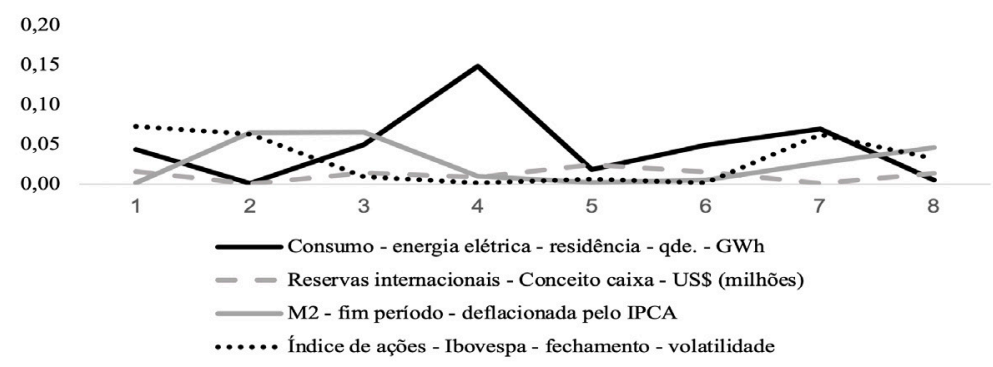

Fonte: Elaboração própria.

Como pode ser observado, as variáveis que se destacam dentre as analisadas como indicadores líderes na análise in sample são o crescimento da oferta de moeda e a volatilidade do índice Ibovespa. Entretanto, a análise out of sample indica uma perda de poder preditivo para as duas variáveis. Desse modo, não é possível concluir que a utilização dessas variáveis para modelos de previsão de médio prazo seja proveitosa. Esse resultado mostra um gap na literatura para a economia brasileira de modelos probit, de modo que futuros trabalhos devem propor outras variáveis que possam ser utilizadas com defasagens maiores. Como mencionado, 
a variável mais utilizada na literatura de outros países é o spread entre as taxas de juros de curto e longo prazo, de modo que, quando uma amostra de dados suficientemente grande estiver disponível, uma análise dessa variável deve ser feita para testar seu poder preditivo na economia brasileira.

Para o consumo de energia elétrica residencial, o pseudo $R^{2}$ apresenta o seu maior valor para previsões quatro trimestres à frente na análise out of sample. Esse resultado condiz com a literatura sobre o tema, uma vez que tanto Oliveira (2016), quanto Chauvet e Morais (2010) utilizam a variáveis do setor energético para construir modelos de médio prazo.

\section{Considerações Finais}

O presente trabalho buscou comparar a capacidade preditiva, a partir da construção do pseudo $R^{2}$, de diferentes variáveis para recessões na economia brasileira no período de 1997 a 2017. Além disso, o artigo tece comparações com outros trabalhos aplicados à economia brasileira sobre o tema.

A partir da estimação dos modelos econométricos, foi possível identificar variáveis que refletem a situação fiscal do país como potenciais candidatos para compor modelos probabilísticos para previsões de recessões no curto prazo, com destaque para a dívida fiscal líquida do setor público e para a dívida total líquida de estados e municípios como porcentagem do PIB. Outra variável, inédita nesse tipo de análise, que mostrou uma performance relativamente boa para modelos de curto prazo é o índice de preço internacional das commodities. Além disso, as variáveis financeiras risco-país e retorno do índice Ibovespa apresentam pseudo $R^{2}$ relativamente altos nas análises in e out of sample para horizontes de curto prazo.

Quanto às variáveis que buscam prever períodos de recessão com horizontes mais distantes, primeiramente foram testadas as séries utilizadas no trabalho de Chauvet e Morais (2010) em modelos probit de médio prazo, como mudanças na balança comercial, consumo de energia elétrica residencial, estoque de veículos comerciais leves, preço das importações e estoque de ônibus produzidos. Entretanto, tanto mudanças na balança comercial quanto o estoque de ônibus produzidos não apresentam relevância estatística para nenhum horizonte de tempo, e as outras variáveis mostraram um poder preditivo relativamente baixo. Dessa forma, foi proposta a utilização de outras três variáveis: a variação nas reservas internacionais, para capturar a política cambial; a taxa de crescimento da oferta de moeda M2 deflacionada pelo IPCA, para efeitos de política monetária; e a volatilidade do índice Ibovespa, para efeitos de expectativas dos agentes financeiros.

Os resultados encontrados indicaram que tanto a taxa de crescimento da oferta monetária quanto a volatilidade do índice Ibovespa são as variáveis de médio prazo que apresentaram as melhores performances na análise in sample. Entre- 
tanto, a estimativa out of sample mostra uma significativa perda de poder preditivo dessas variáveis, frustrando a tentativa de encontrar variáveis que pudessem ser utilizadas em modelos probit com maiores defasagens.

A principal limitação deste artigo se refere à impossibilidade de se testar o spread da taxa de juros de curto e longo prazo como indicador líder, consequência da inexistência de uma amostra suficientemente grande de dados para estimação de um modelo consistente.

Novas contribuições no tema devem emergir com a ampliação do espaço amostral para as variáveis analisadas neste artigo, além da inclusão de variáveis que atualmente não possuem um número de observações suficiente para a estimação dos modelos probit. Dentre elas, o spread da taxa de juros de curto e longo prazos e variáveis que capturam o sentimento de consumidores e produtores quanto ao ambiente de negócios no país, como, por exemplo, o purchasing manager index (PMI). Além disso, novas contribuições podem surgir a partir da estimação de modelos probit simples e dinâmicos utilizando-se as variáveis apresentadas com a realização de back test para checar acertos e erros de previsão de potenciais modelos. Por fim, há espaço para trabalhos que busquem encontrar variáveis que apresentem uma boa performance para previsões de horizontes mais longos.

Vale ressaltar que a utilização de modelos probit não deve substituir modelos macroeconômicos mais complexos, servindo como uma ferramenta adicional para previsões sobre o futuro estado da atividade econômica do país.

\section{Referências}

ABREU, M. P. (ed.). A ordem do progresso: dois séculos de política econômica no Brasil. Rio de Janeiro: Elsevier, 2015.

BISMANS, F.; MAJETTI, R. Forecasting recessions using financial variables: the French case. Empirical Economics, v. 44, n. 2, p. 419-433, 2013.

BRASIL. Instituto de Pesquisa Econômica Aplicada (IPEA). [2018]. Disponível em: ttp://www. ipeadata.gov.br/Default.aspx. Acesso em: abr. 2018.

CHAUVET, M. The Brazilian business and growth cycles. Revista Brasileira de Economia, v. 56, n. 1, p. 75-106, 2002.

CHAUVET, M.; MORAIS, I. Predicting recessions in Brazil. In: COLLOQUIUM ON MODERN TOOLS FOR BUSINESS CYCLE ANALYSIS: "THE LESSONS FROM GLOBAL ECONOMIC CRISIS". 6., 2010, Luxembourg. Proceedings [...]. Luxembourg: Eurostat; Florence: European University Institute, 2010.

CHAUVET, M.; POTTER, S. Forecasting recessions using the yield curve. Journal of Forecasting, v. 24, n. 2, p. 77-103, 2005. 
FUNDAÇÃO GETULIO VARGAS (FGV). Comitê de Datação de Ciclos Econômicos (CODACE). Out. 2017. Disponível em: https://portalibre.fgv.br/sites/default/files/2020-03/comite-de-data_ode-ciclos-econ_micos-comunicado-de-30_10_2017-_1_.pdf. Acesso em: 1 abr. 2018.

DEL NEGRO, A. Turn, turn, turn: predicting turning points in economic activity Economic Review - Federal Reserve Bank of Atlanta, v. 86, Q2, 2001.

DUARTE, A. J.; ISSLER, J. V.; SPACOV, A. Indicadores coincidentes de atividade econômica e uma cronologia de recessões para o Brasil. FGV EPGE Economics Working Papers, n. 527, 2004.

ESTRELLA, A.; HARDOUVELIS, G.A. The term structure as a predictor of real economic activity. The Journal of Finance, v. 46, n. 2, p. 555-576, 1991.

ESTRELLA, A.; MISHKIN, F. S. Predicting U.S. recessions: financial variables as leading indicators. Review of Economics and Statistics, v. 80, n. 1, p. 45-61, 1998.

GIAMBIAGI, F.; VILLELA, A.; BARROS DE CASTRO, L.; HERMANN, J. (org.). Economia brasileira contemporânea. 2. ed. Rio de Janeiro: Campus, , 2011.

GRUBER, J. Public finance and public policy. New York: Worth Publishers, 2013, p. 304-305.

GUIMARÃES, B. A riqueza da nação no século XXI. São Paulo: Bei Comunicação, 2015.

HAO, L.; NG, E. C. Y. Predicting Canadian recessions using dynamic probit modelling approaches. The Canadian Journal of Economics/Reuue Canadienne D'Économique, v. 44, n. 4, p. 1297-1330, 2011.

IBGE, Instituto Brasileiro de Geografia e Estatística. Sistema de Contas Nacionais Trimestrais - SCNT. Disponível em: <https://www.ibge.gov.br/estatisticas/economicas/ contas-nacionais/9300-contas-nacionais-trimestrais.html $?=\mathcal{E} \mathrm{t}=$ series-historicas $\mathcal{Z} u t m$ source $=$ landingE्(utm_medium $=$ explicaEJutm_campaign $=$ pib\# evolucao-taxa $>$. Acesso em: 1 abr. 2018.

LOPES, L.; CHAUVET, M.; LIMA, J. Trend-cycle decomposition of the Brazilian GDP: new facts for the period between 1947 and 2012. In: ENCONTRO NACIONAL DE ECONOMIA, 15., 2017, Natal. Proceedings [...]. Natal: ANPEC, 2017.

MESQUITA, M. A política econômica do governo Dilma: a volta do experimentalismo. In: CENTRO DE DEBATE DE POLÍTICAS PÚBLICAS. Sob a luz do sol, uma agenda para o Brasil. São Paulo: CDPP, 2014.

OLIVEIRA, F. N. Financial and real sector leading indicators of recessions in Brazil using probabilistic models. Revista Brasileira de Economia, v. 70, n. 3, p. 337-355, 2016.

PASTORE, A. C.; PINOTTI, M. C. Inflação e estabilização: algumas lições da I experiência brasileira. Revista Brasileira de Economia, v. 53, n. 1, p. 3-40, 1999. Disponível em: http:// bibliotecadigital.fgv.br/ojs/index.php/rbe/article/view/747. Acesso em: 30 abr. 2018.

PIRES, J. C. L. et al. O cenário macroeconômico e as condições de oferta de energia elétrica no Brasil. Textos para Discussão BNDES, n. 85, 2001. 
Apêndice A

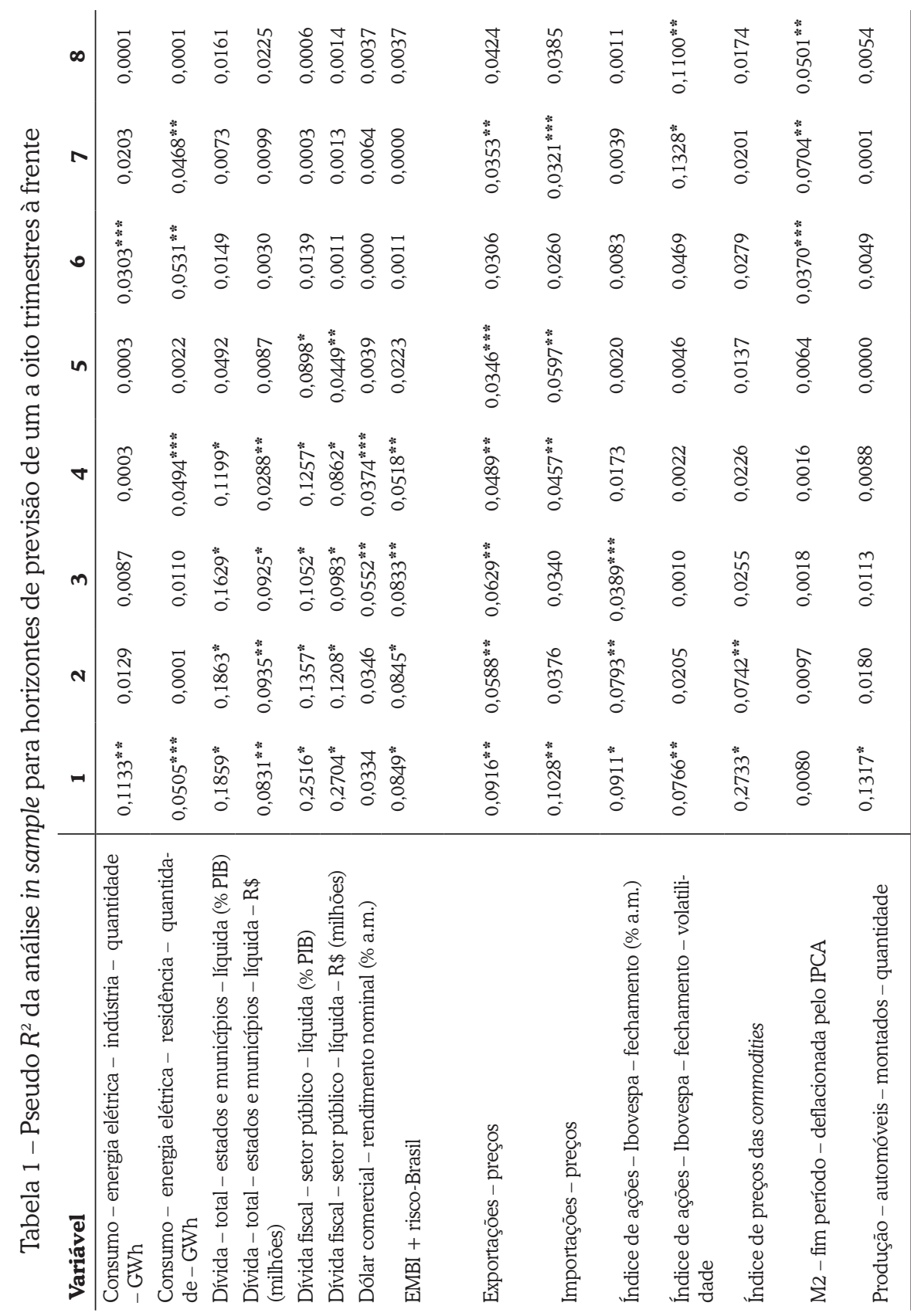




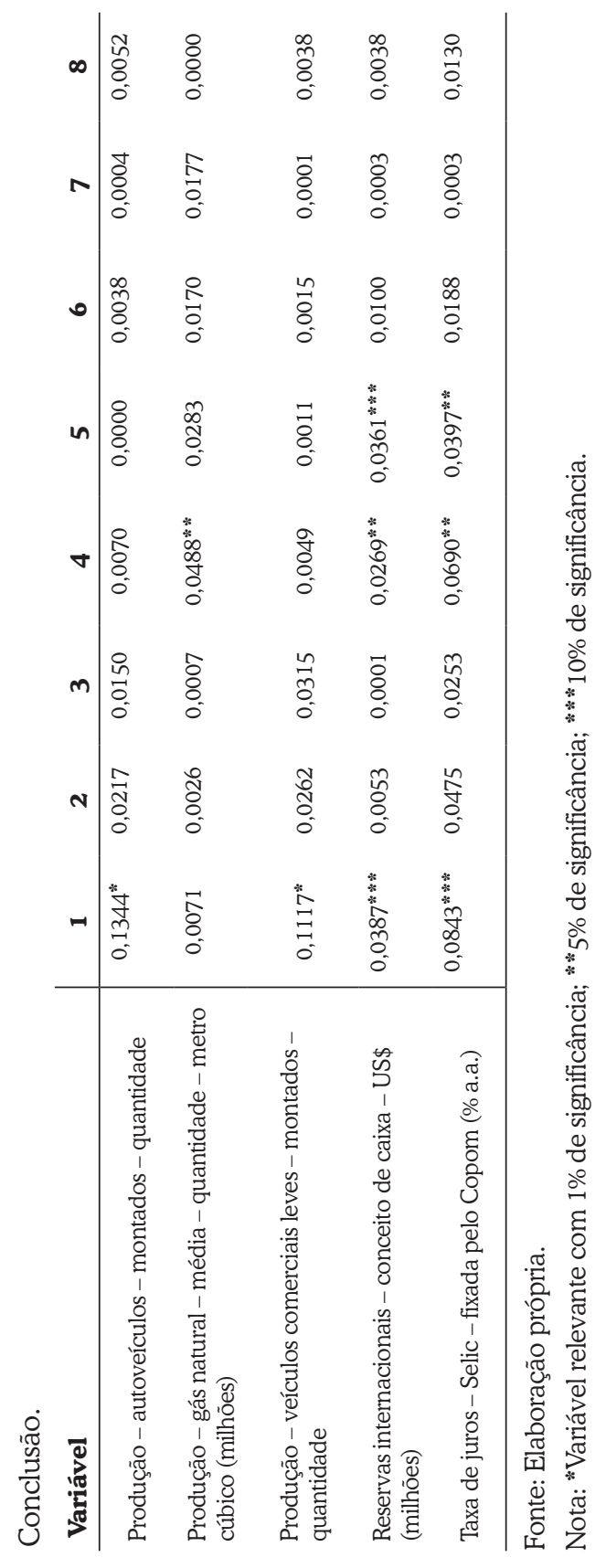




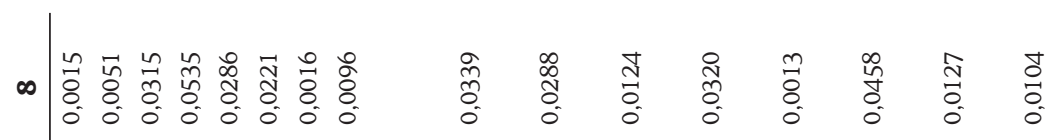

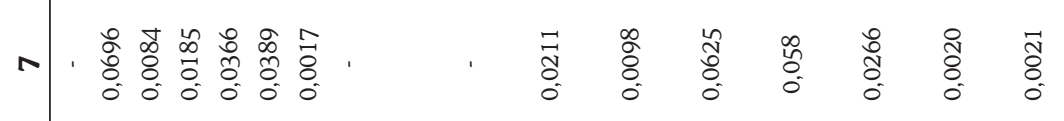

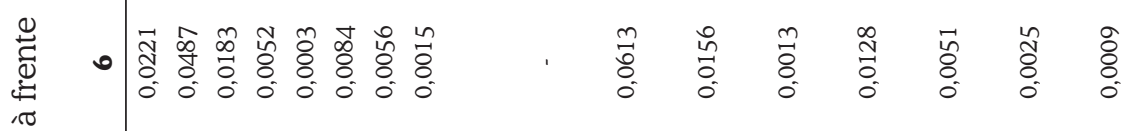

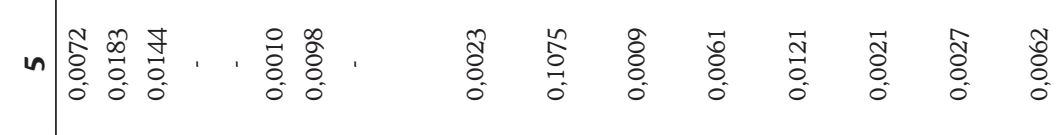

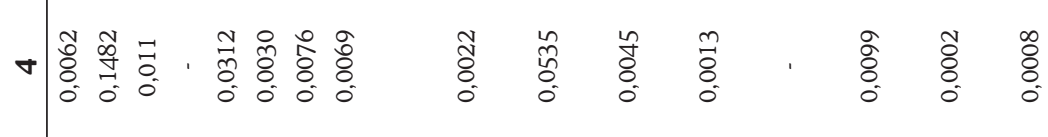

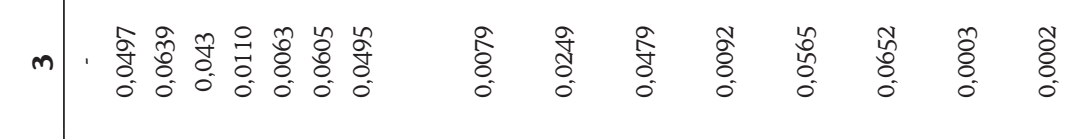

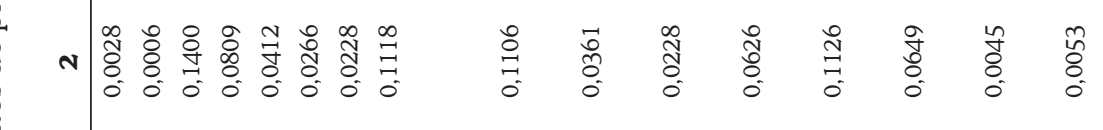

กับ

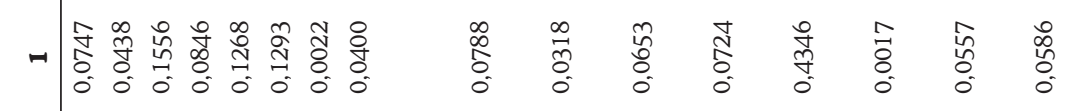

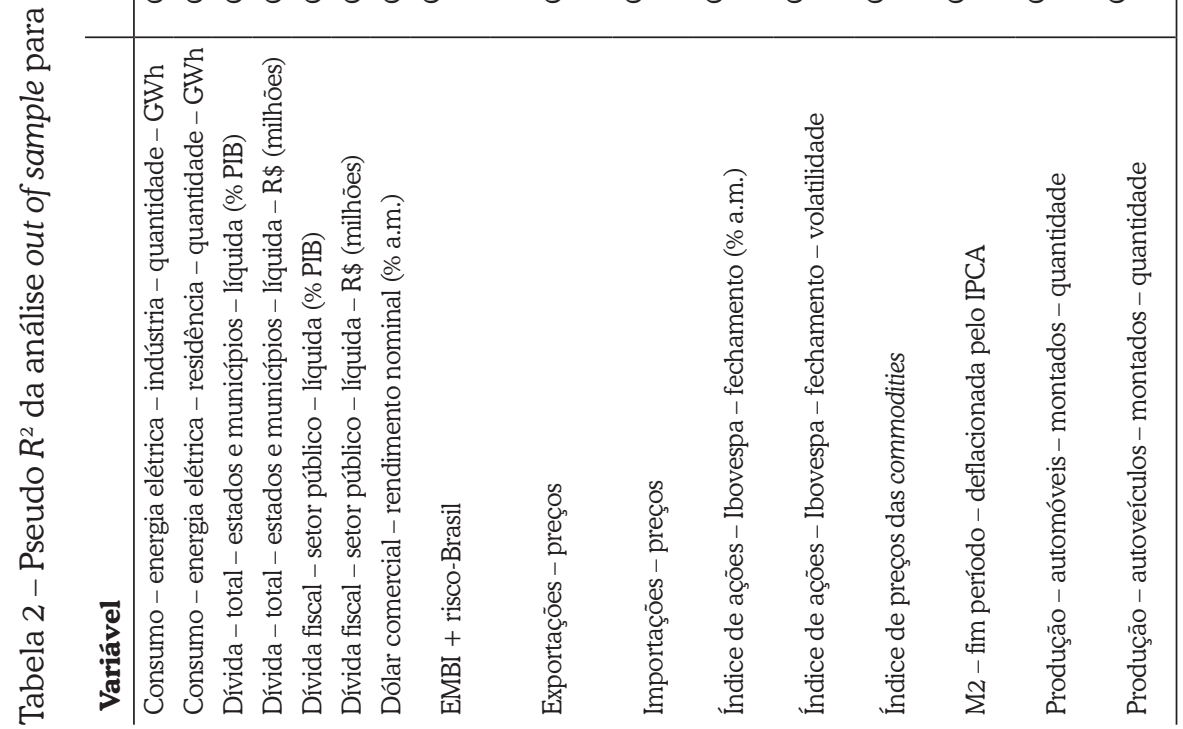




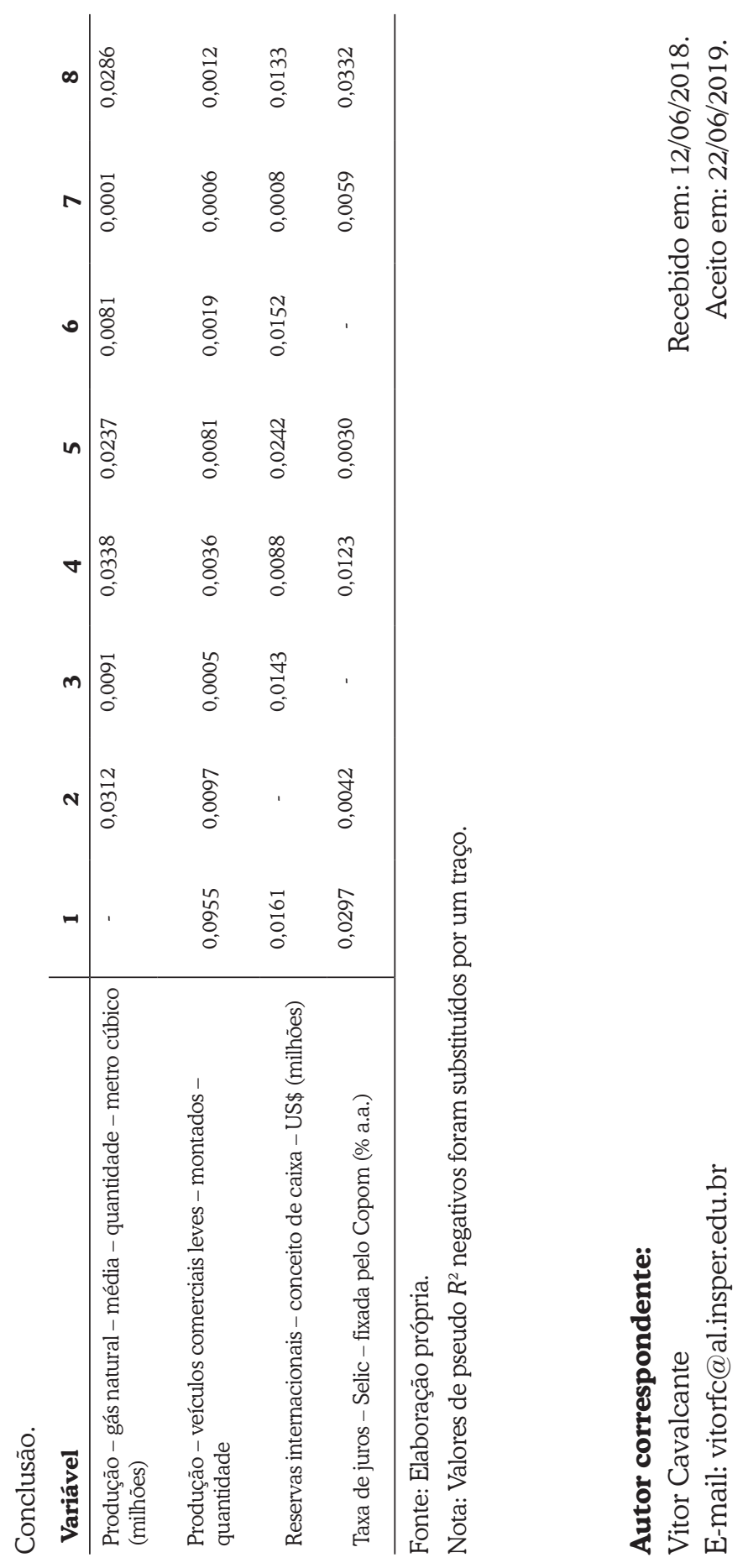

